\title{
Utilizing Calibrated GPS Reflected Signals to Estimate Soil Reflectivity and Dielectric Constant: Results from SMEX02
}

\author{
Stephen J. Katzberg*, Omar Torres*, Michael S. Grant* and Dallas Masters ${ }^{\dagger}$ \\ *NASA-Langley Research Center Hampton, VA 23681 \\ ${ }^{\dagger}$ University of Colorado, Boulder, CO 80309
}

\begin{abstract}
Extensive reflected GPS data was collected using a GPS reflectometer installed on an HC130 aircraft during the Soil Moisture Experiment 2002 (SMEX02) near Ames, lowa. At the same time, widespread surface truth data was acquired in the form of point soil moisture profiles, areal sampling of near-surface soil moisture, total green biomass and precipitation history, among others. Previously, there have been no reported efforts to calibrate reflected GPS data sets acquired over land. This paper reports the results of two approaches to calibration of the data that yield consistent results. It is shown that estimating the strength of the reflected signals by either (1) assuming an approximately specular surface reflection or (2) inferring the surface slope probability density and associated normalization constants give essentially the same results for the conditions encountered in SMEX02. The corrected data is converted to surface reflectivity and then to dielectric constant as a test of the calibration approaches. Utilizing the extensive in-situ soil moisture related data this paper also presents the results of comparing the GPS-inferred relative dielectric constant with the Wang-Schmugge model frequently used to relate volume moisture content to dielectric constant. It is shown that the calibrated GPS reflectivity estimates follow the expected dependence of permittivity with volume moisture, but with the following qualification: The soil moisture value governing the reflectivity appears to come from only the top 1-2 centimeters of soil, a result consistent with results found for other microwave techniques operating at L-band. Nevertheless, the experimentally derived dielectric constant is generally lower than predicted. Possible explanations are presented to explain this result.
\end{abstract}

\section{Introduction}

Soil water content, or soil moisture, is a key component of the water cycle. It directly influences the amount of evaporation, infiltration, runoff, and the amount of water uptake by plants [Koster et al., 2004]. Soil moisture creates energy fluxes between the land and the atmosphere that induce weather systems that can affect large populated areas. Moreover, the accurate monitoring of soil moisture serves as a factor in hydrological and vegetation monitoring [Kerr et al., 2001] and for better seasonal forecasting [Koster et al., 2004].

The impact of soil moisture on atmospheric processes, such as rain, has been studied over the years, but these studies are limited by the lack of direct and global observational data and must recur to numerical models [Koster et al., 
2004; Kerr et al., 2001]. The importance of an accurate widespread soil moisture measurement is well recognized within the scientific community [see for instance O'Neil et al., 1993; Wood et al., 1993; Jackson and Schmugge, 1992; Kostov et al., 1991; Jackson and O'Neil, 1988; Njoku and Kong, 1977]. However, the monitoring of large area surface water resources is generally impractical via in situ observations because of the large number of sites required and the high cost of monitoring equipment. Air (or space) borne remote sensors using either thermal emission or radar backscatter are now accepted as a viable solution to the problem and considerable effort has gone into the development and validation of these tools [see for instance Narayan et al., 2004; Holmes, 2003; Njoku, 1999; Le Vine et al., 1994].

The spectral band that works best for soil moisture monitoring lies within the LBand (1-2 GHz) because at these frequencies (1) the atmosphere is effectively transparent, (2) vegetation is semi-transparent allowing observations of the surface, (3) the microwave measurement is strongly dependent on soil moisture, and (4) the measurements are independent of solar illumination [Jackson and Schmugge, 1992]. However, microwave measurements from air or space have been limited by the large antennas required to obtain reasonable spatial resolution. For instance, JPL's PALS instrument, which is regarded as one of the best instruments to remotely sense soil moisture, uses two horn antennas half a meter and one meter in diameter each [Wilson, et al. 2001] and provides a $3 \mathrm{~dB}$ footprint on the surface of about $330 \times 470 \mathrm{~m}$ at $\sim 1100 \mathrm{~m}$ above the surface [Narayan, et al. 2004]. Aperture synthesis is used by some complex systems in an attempt to reduce the physical size of the antenna required to provide acceptable resolutions. Such is the case of ESTAR, which is a hybrid real-andsynthetic radiometer that uses five stick antennas with a linear array of eight horizontally polarized dipoles each [Le Vine et al., 1994] providing higher resolution: $200 \mathrm{~m}$ grids at $2200 \mathrm{~m}$ nominal altitude [Jackson et al., 1994].

The GPS reflectometer developed at NASA's Langley Research Center makes use of the nearly thirty satellites that transmit a continuous range-coded signal around the world. The structure of the signal is such that the correlation properties of the pseudorandom noise (PRN) GPS signal can serve as a remote sensing measurement instead of the conventional measure of received power [Katzberg and Garrison, 1996]. The reflectometer then consists of only a receiver capable of decoding the civil segment of the GPS, which is transmitted at a center frequency of $1.57542 \mathrm{GHz}$. As a consequence of the range coded signal, the required antenna size can be very small compared to antennas employed by other techniques.

The reflected PRN code is collected from signals that reflect from the surface of the earth in a forward-scattering configuration analogous to bistatic radar [Katzberg and Garrison, 1996]. The signals lie within the most favorable spectral band for soil moisture sensing, and the system has demonstrated the capability to obtain signal to noise ratios high enough to sense small changes in surface 
reflectivity [Masters et al., 2004].

The simplicity of the receiver, its light weight, small size, and low power consumption make it attractive for use in many field or flight platforms. Future GPS satellites will carry two new civil signals at $1.2276 \mathrm{GHz}$ (L2) and 1.17642 $\mathrm{GHz}$ (L5) [Hegarty et al., 2000] providing measurement opportunities at three different wavelengths that will provide diverse views of apparent vegetation opacity and soil penetration depth.

This paper presents a follow-on and expansion of earlier work related to the use of GPS reflection remote sensing [Masters, et al.2004] and reports the first calibration of reflected GPS signals and estimation of surface dielectric constant compared to surface truth. The material is divided into six sections. Section 2 describes the experimental setup and the types of data collected. Section 3 provides background on GPS reflections and a description of the factors that influence the performance of the reflected signals. Section 4 describes the processing methods followed to analyze the data and the results obtained. Section 5 provides possible explanations and observations on the performance of the method proposed. Finally, section 6 concludes and two appendices provide details on the Wang-Schmugge numerical model and Theta probe functionality.

\section{Soil Moisture Experiment 2002}

The first experiment to be conducted in which concurrent GPS reflected signals from land and in-situ data were collected occurred during the Soil Moisture Experiment of 2002 (referred to as SMEX02.) The experiment was conducted in lowa during the months of June and July focusing on intensively sampled sites in the Walnut Creek region south of Ames. The experiment included the participation of many scientists and technicians from various agencies and universities that collected data suitable to support the verification and validation of soil moisture and brightness temperature algorithms from the EOS Aqua Advanced Microwave Scanning Radiometer (AMSR-E) [Njoku et al., 2004]. The experiment included performance of new instrument technologies, including the GPS reflectometer, with potential applications to remote sensing of the global hydrological cycle. (For a detailed description of the experiment the reader is directed to an earlier special issue of this publication [Njoku, et al., 2004])

As a result of the experiment, high quality, comprehensive in-situ data sets were collected to serve as part of the basis for developmental and current algorithms that study and model land-atmosphere interactions. Some of the data sets collected (only those relevant to the study presented in this paper) are summarized in the reminder of this section. For the complete data acquired in SMEX02 the reader is referred to the National Snow and Ice Data Center (NSIDC) web site at http://nsidc.org/data/amsr_validation/soil_moisture/. 


\subsection{In-Situ Measurements}

The in-situ data collected over the Walnut Creek region included soil texture, soil moisture, surface roughness, vegetation water content, and precipitation. The sampling region contained thirty-two sites; twenty one of the sites were cornfields, ten were soybean fields, and one was a grass field. However, the data collected by the GPS reflectometer did not overlap with the grass field. The measurements collected coincided with the aircraft-based microwave investigations conducted between 0900 and 1200 hours local time each day that data were acquired.

\subsubsection{Soil moisture samples}

Soil moisture was measured across the region using different techniques such as theta probe measurements, core sampling, and time delay reflectometry (TDR;) a summary of soil moisture measurements is presented in Table 1 . The primary measurement of soil moisture was performed using Theta probes (See Appendix A for a more detailed explanation of Theta probes.) The device provided an average measurement of soil moisture from the top five centimeters of soil. The average acreage of the WC sites accommodated from seven to fourteen Theta probe measurements 100 meters apart.

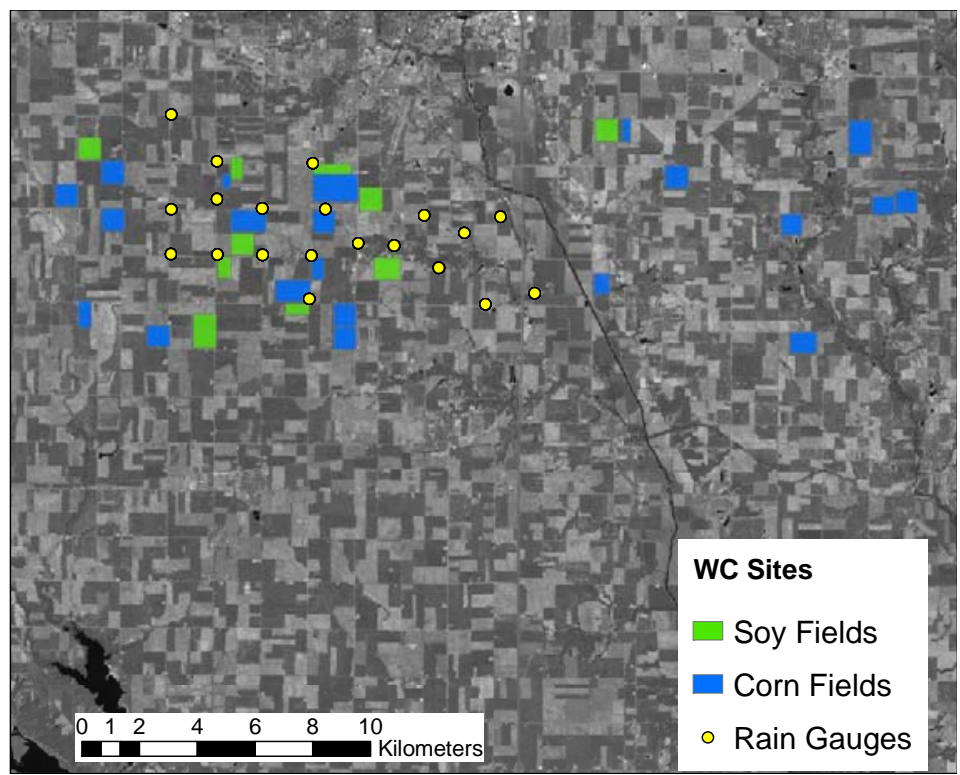

Figure 1. The Walnut Creek sampling region included twenty-one cornfields and ten soybean fields. Precipitation data was also collected across the region.

In addition, gravimetric soil moisture (GSM) was measured at four WC fields using sliced core sampling techniques. The four sites, WC 15 (corn,) WC 16 (soybeans,) WC 23 (soybeans,) and WC 24 (corn,) contained four samples each from which the bulk density of the soil was extracted. Each sample was cut into 
six 1-cm slices to determine the GSM for each layer. The volumetric soil moisture at each layer was obtained by multiplying bulk density by the GSM.

The soil moisture profile as a function of depth was also obtained using TDR from the four special WC sites mentioned. TDR measurements were performed every fifteen minutes and provided soil moisture measurements at two, five, ten, fifteen, twenty, and thirty centimeters depth. The sliced core and TDR samples represent the highest resolution, most reliable, soil moisture profiles taken during the experiment.

Both TDR and sliced core soil moisture sampling techniques showed large differences between the soil moisture at the surface and at thirty centimeters depth. Core sample measurements showed values as low as $0.67 \%$ at the surface [Laymon, 2003] while TDR showed soil moisture values up to $30 \%$ percent at depths of one-third meter.

\begin{tabular}{|c|c|c|c|c|}
\hline $\begin{array}{c}\text { Type of } \\
\text { Measurement }\end{array}$ & Technique & Locations & $\begin{array}{c}\text { Frequency of } \\
\text { Measurement }\end{array}$ & Depth(s) \\
\hline $\begin{array}{c}\text { Gravimetric } \\
\text { profile }\end{array}$ & $\begin{array}{c}\text { Core sample, } \\
\text { weighing }\end{array}$ & lowa regional & Daily & Top 1, $2 \mathrm{~cm}$ \\
\hline $\begin{array}{c}\text { Volumetric } \\
\text { profile }\end{array}$ & Theta Probe & lowa regional & Daily & Top 0-6 cm average \\
\hline $\begin{array}{c}\text { Volumetric } \\
\text { profile }\end{array}$ & $\begin{array}{c}\text { Time Delay } \\
\text { Reflectometer }\end{array}$ & WC 15, 16, 23, 24 & $\begin{array}{c}\text { Continuously } \\
\text { every 15 } \\
\text { minutes }\end{array}$ & $\begin{array}{c}2,5,10,15,20,30 \\
\mathrm{~cm}\end{array}$ \\
\hline $\begin{array}{c}\text { Volumetric } \\
\text { profile }\end{array}$ & Theta Probe & Multiple in all \\
WC's & Daily & Top 0-6 cm average \\
\hline $\begin{array}{c}\text { Gravimetric } \\
\text { profile }\end{array}$ & $\begin{array}{c}\text { Sliced core } \\
\text { sample, } \\
\text { weighing }\end{array}$ & WC 15, 16, 23, 24 & $\begin{array}{c}\text { Daily (some } \\
\text { missing days) }\end{array}$ & $\begin{array}{c}\text { Average in top 1, 2, } \\
3,4,5,6 \mathrm{~cm} \text { slices }\end{array}$ \\
\hline
\end{tabular}

Table 1. Soil moisture in-situ measurements taken during SMEX02.

\subsubsection{Plant biomass measurements}

At the outset of the SMEX02 period, corn was in the early stages of growth and most soybean fields were essentially bare soil. By the end of the experiment, corn biomass ranged between three and four kilograms per square meter, while soybeans had a biomass of less than one kilogram per square meter. Data describing the time evolution of vegetation, canopy height, and green biomass were collected several times during the experiment for all sites. Each biomass sample was separated into leaf and stem to determine the water content of the individual components. The plant's water content and height were referenced to a cross-sectional area of one square meter to determine the total volume moisture content.

Sampling of green biomass was done on a regular basis, but not daily. Thus, 
when flights occurred between sampling times, a linear interpolation for biomass moisture content was used. For sampling times close to the over-flights, the sampled value was used without interpolation. However, if over-flights occurred after the last biomass sampling, the GPS data were not used to avoid assumptions in biomass data extrapolation.

\subsubsection{Precipitation history}

Twenty rain gauge sampling locations scattered throughout the Walnut Creek area, but not collocated with the sampling sites (see Figure 1,) collected rain information during SMEX02. Before the experiment, there was a period of over ten days without any precipitation. The first rain event occurred on July $4^{\text {th }}$ in the afternoon, then on July $5^{\text {th }}$, July $6^{\text {th }}$ and July $7^{\text {th }}$. Subsequent flights were flown the day after significant rain and before afternoon rain for July $5^{\text {th }}$ and July $6^{\text {th }}$. The rain events are summarized in Table 2, although it should be noted that the precipitation was not uniform across the region: During the period reported here, there was considerably more rain on the western part of Walnut Creek while there was little or no rain on the eastern part. The difference augmented soil moisture on one part of the experimental area while remaining almost constant on the other.

\begin{tabular}{|c|c|c|}
\hline Flight Date & Rain before flight & Rain after flight \\
\hline \hline $7 / 1 / 02$ & None for over ten days & None \\
\hline $7 / 5 / 02$ & Yes & Yes \\
\hline $7 / 6 / 02$ & Yes & Yes \\
\hline $7 / 8 / 02$ & Yes (very small amount) & N/A \\
\hline
\end{tabular}

Table 2. Summary of the precipitation events during SMEX02.

\subsubsection{Surface roughness measurements}

Soil roughness was measured at four sampling locations in each WC field: a picture was taken at every sampling location using as background a two centimeter grid board one meter in length. The pictures were input into a commercial program called SigmaScan pro 4 to obtain the surface slopes, root mean square height, correlation length, and the correlation function of the surface.

\subsection{GPS in SMEX02}

The GPS reflectometer was flown on the NCAR HC-130 aircraft with the JPL PALS instrument. The aircraft flew at an altitude of approximately $1100 \mathrm{~m}$ above the surface at an average speed of $75 \mathrm{~m} / \mathrm{s}$ while the reflectometer recorded data at a rate of $10 \mathrm{~Hz}$. Reflected power was recorded during flights on June $25^{\text {th }}$, 
June $27^{\text {th }}$, July $1^{\text {st }}$, July $5^{\text {th }}$, July $6^{\text {th }}$, and July $8^{\text {th }}, 2002$ and at the same time frame than the in-situ data.

During the flights, the reflectometer utilized the highest elevation satellite in view so that the average elevation angle was generally above sixty-five degrees (twenty-five degrees incidence angle.) Each flight lasted approximately two hours, which resulted in two to three satellites being utilized.

The data collected corresponded to satellites with PRN 10, 17, 23, and 24. However, signal from the satellite with PRN 17 was neglected since the satellite was officially out of service during this period and the data could not be considered reliable. Each satellite's reflected data were averaged across each field for every day to estimate the reflected power from each WC field. Furthermore, the average reflected power from each field was compared to the daily average soil moisture reading from that particular site for comparison.

\section{GPS Remote Sensing}

\subsection{Background on GPS Reflections}

A complete discussion of the GPS technical details can be found in Spilker and Parkinson (1996), however some characteristics important to understanding the GPS reflection technique are summarized in the following paragraphs.

The GPS satellite constellation consists of 24 satellites that orbit the earth at a mean altitude of $20,126.61 \mathrm{Km}$ over the earth's equatorial radius. The satellite orbits are designed so that at any point on the Earth there are at least four satellites in view at all times for three-dimensional navigation. The signals transmitted by all satellites are range-coded at the same frequency, but each has its own unique, separable signature. The carrier frequencies of GPS are capable of traveling through clouds, rain, and fog, but strongly reflect from water bodies such as oceans, lakes and soil moisture. The signals change from right-hand circularly polarized (RHCP) to predominantly left-hand circular polarized (LHCP) at reflection and occasionally interfere with the direct signals causing erroneous position estimates [See for example, Auber et al., 1994]. While the reflected signal is generally weaker than the incoming signal, the residual strength of these signals still provides opportunity for remote sensing applications.

The GPS signal structure is based upon the use of code division multiplexing in which a common carrier frequency $(1.575 \mathrm{GHz}$ or $1.227 \mathrm{GHz})$ is used with a binary, bi-phase pseudorandom noise (PRN) code modulation. For the GPS signals accessible by the civilian community, the PRN signals are unique Gold codes 1023 bits in length (also referred to as code-chips) and repeat in one millisecond (each chip is either \pm 1 with a duration of $1 / 1023$ milliseconds.) Since the speed of light is close to 299 meters per microsecond, each chip represents a 
physical length of approximately 292.3 meters.

Each satellite transmits a PRN code that is different from the PRN codes transmitted by all other satellites. These codes are orthogonal to each other in the sense that one code multiplied by a different member of the set and the result integrated for a certain time, a nearly zero value is obtained. If the code is multiplied by a perfectly phased copy of itself, a very high value results from the integration. Thus, a single RF channel can be used by a GPS receiver to detect and track several satellites at the same time with the signal from each satellite isolated by simply processing the matching code for the desired satellite. Also, impressed on the PRN code is a second, slowly changing, bi-phase signal that carries additional information required to perform position location (e.g. satellite ephemeris data.)

GPS receivers generate exact replicas of all the PRN codes transmitted by the satellites. Any GPS receiver must correlate each received signal with its exact replica to extract signal timing and recover navigation data for the desired satellite. The correlation process consists of comparing the received signal with the replica at different phases by multiplying the signals and integrating the product over the 1023 code chip interval. When the received signal is in perfect phase with the receiver's replica, the multiplication and integration of these yields the product of the bi-phase amplitudes times the code time interval multiplied by the code repetition period. If the two codes are shifted from perfect synchronism and integrated, the result decreases to a low value. This maximum-value-downto-minimum value phase shifting happens in one code-chip since even a shifted replica of a code is orthogonal to its twin in the same way as another member of the code set is.

The integration result as a function of phase shift is a linear decrease in signal level for either positive or negative shift from perfect overlap and is called the "lambda" function from its resemblance to the capital Greek letter $\Lambda$. For many applications, it is convenient to square the resulting cross-correlation to obtain a positive definite result. This squaring of the lambda-function gives what is called the "lambda squared" function. The amplitude of the "lambda squared" function is proportional to the product of the amplitudes of the received signal and the receiver's replica. While the internal receiver replica code has a fixed, arbitrarily set amplitude, the reflected signal is proportional to the electric field at the antenna. Thus, the cross-correlation and squaring process in the receiver produces a result proportional to reflected signal power. An example of the "lambda squared" waveform from SMEX02 is shown in Figure 2a.

\subsubsection{Surface Roughness effects}

Now consider the result of the GPS signal striking a smooth earth; the GPS 
signal travels the shortest distance to the GPS receiving antenna when striking a point in the plane of incidence for which the angle of incidence equals the angle of reflection (Figure 2b.) Any other point on the surface yields a longer distance from satellite to receiver and results in a phase shift in the correlation process greater than that corresponding to the signal reflecting at the specular point.

The excess distance traveled by the signal reflecting at the specular point (compared to the distance traveled by a signal coming directly to the GPS receiving antenna from the satellite) can be expressed as:

$$
\text { (1) } \delta=2 \cdot h \cdot \sin \gamma
$$

Where $h$ is the height of the receiver above the surface and $\gamma$ is the elevation angle for the signal source (GPS satellite.)

The locus of points with equal excess path length away from the specular point can be shown to be [Beckman and Spizzichino, 1963, Katzberg and Garrison, 1996] a family of ellipses whose center is at:

$$
\text { (2) } \begin{aligned}
& S_{x}=h \cdot \cot \gamma \\
& S_{y}=0 .
\end{aligned}
$$

Where $S_{x}$ is the location of the ellipse center on the surface of the earth in the direction of the satellite $S_{y}$ is the center in the direction normal to the satellitespecular-point-antenna line

The extent of the ellipse projected onto the surface can be expressed in terms of its semi-major axis $a$ (aligned along the direction to the GPS satellite) and its semi-minor axis $b$ :

$$
\text { (3) } \begin{aligned}
a & =\frac{\sqrt{2 h c \tau_{2} \sin \gamma}}{\sin ^{2} \gamma} \\
b & =\frac{\sqrt{2 h c \tau_{1} \sin \gamma}}{\sin \gamma}
\end{aligned}
$$

Where $c$ is the speed of light and $\tau$ is the delay of the reflected path in excess of that from the specular point. An illustration of the ellipses is shown in Figure 2b.

If the surface is flat over an expanse of several wavelengths, the signal appears to reflect from an ellipse whose size is set by the wavelength $\lambda$. A typical approximation is to substitute the factor $\lambda / 2$ for the product $c \tau$ to give the diameter of this ellipse, which is the first "Fresnel zone." The result may be thought of as giving the ultimate resolution "pixel" for the GPS technique. 
On the other hand, if the surface is not smooth, slopes may exist with the proper orientation to redirect the incoming radiation to the receiving antenna from sites away from the specular point. This scattered power can still be strong enough to produce a detectable signal in the receiver. These signals, however, reach the receiver after a longer delay than the signal arising from the area near the specular point. Signals arising from elemental areas near any particular elliptical delay ring on the surface produce signals from each scattering center with the same code delay. These signals with similar delays carry modulation that is a replica of the incoming pseudo-random-noise code but each still has individual carrier phase.

Under the assumption that natural scenes are composed of independently phased scatterers, the resulting composite signal is stochastic. Consequently, the expectation of the squared cross-correlation is the sum of all the scattered power along that ring of the ellipse corresponding to a particular delay, with the lambda-squared function as weighting factor. In this way the cross-correlation and squaring process produces a result that is sensitive to power only around a certain ellipse corresponding to the selected delay. The width of the active region on the surface corresponding to a particular delay is the area between the ellipses corresponding to plus or minus one code chip on the surface. The sensitivity profile across that enclosed area is that of the lambda-squared function projected onto the surface.

Stepping through a range of delays in the receiver is thus seen to be a method of scanning the surface via expanding concentric elliptical rings. Since each ellipse is characterized by the same time delay, the recorded power in the receiver represents a power versus delay.

The existence in each ellipse of scatterers properly oriented to redirect signal to the antenna is controlled by the two-dimensional slope probability function. In simple terms, the further away from the specular point, the higher the slopes must be to redirect the signal in the right direction. The fraction of scatterers in the elemental ellipse with the required slopes is described by the 2-d slope probability density.

The effects just described may be thought of as the influence of surface roughness on the detected signal. The received signal from each scatterer is also affected by the material characteristics of each surface element. The signal strength associated with each scatterer depends upon the modulus squared of the Fresnel reflection coefficient (power reflectivity) of each surface slope element that also depends on the element scattering angles.

Finally, the power signal in the receiver at any delay is the result of summing up all the scattered power from the elemental surface ellipses multiplied by a lambda-squared function corresponding to that delay. This process may be 
described as a convolution of the lambda-squared function with the surface slope probability function evaluated along elliptical loci of constant delay. It is worth noting here that whatever the source of scattering from the surface, topographic or biomass, or its scattering angles, the reflectometer is capable of recording both.

Some limiting forms: If the slope probability function is nearly a delta function (very smooth surface) the power versus delay is virtually identical to the lambdasquared function. If the slope probability function exists over a broad range of delays, the power versus delay resembles the 2-d slope probability function.


Figure 2. The cross-correlation of the PRN code at different delays gives rise to the lambda-square profile, which is a function of range on the reflecting surface. The sensitivity profile corresponds to an elliptical area on the surface and extends one-half code chip at each delay bin.

For either rough or smooth cases, the forward-scattered (bistatic) configuration of the GPS remote sensing technique is seen to be complementary to backscatter measurements acquired by conventional monostatic radar (scatterometers) and to microwave radiometers, which measure surface emission for studying surface characteristics.

\subsubsection{Vegetation Canopy Effects}

Further scattering and attenuation occurs when the signal path includes at least partial transit through a vegetation canopy. Models have been developed to account for the effects caused by both stalks and leaves [Ulaby, et al., 1986]. According to a model developed by Ulaby et al. (1986) the effect of stalks is negligible at normal incidence and attenuation is caused by the leaf layers. The 
loss factor due to the leaves is expressed as

$$
L_{a}^{\prime}(\vartheta)=\exp \left(\frac{4 \pi}{3 \lambda_{0}} \nu_{l} \varepsilon_{l} h_{l} \sec (\vartheta)\right),
$$

where the variable $v_{l}$ is biomass volume moisture content, $\varepsilon_{l}$ " is the loss component of the dielectric constant of water-containing leaves, $h_{l}$ is the canopy height of the biomass layer, $\lambda_{o}$ is the free space wavelength of the signal, and $\vartheta$ is the incidence angle (the complement of the elevation angle.) Thus the reflected GPS signal is reduced in passing into and out of the vegetation canopy, but the effect can be accounted for with knowledge of the biomass characteristics.

Most surface and vegetation effects on the received signals that interfere with correct estimation of surface reflectivity are associated with the reflected signal. However, the direct signal is subject to the sometimes-serious effects of multipath due to signal reflection from aircraft surfaces. The specific geometry of satellite and aircraft fuselage dictates the interference effects that result in a modification of the detected signal power.

Thus, the proper interpretation of the geological features on the surface must consider the adverse effects on both the direct and reflected signals. Effects introduced by the hardware, such as the relative calibration mismatch between the direct and the reflected channels are explained in the following section.

\subsection{Calibration of the GPS reflectometer}

In order to use the modified GPS receiver as a consistent and accurate reflectometer both the direct and reflected channels must be calibrated. The signal calibration must take into account (1) multipath resulting from aircraft surfaces and simulate what should be a nearly constant source of illumination and (2) the calibration must also take into account the differences in the RF signal chain of the direct (top) and reflected (bottom) channels

At the receiver, the direct and reflected signals follow two virtually identical processing chains. However, the rather simple analog to digital (A/D) system was not originally designed for balanced operation or calibration. The A/D is slightly temperature sensitive while the antenna-connecting cable lengths, preamp gains, noise figures, etc., contribute to a direct-versus-reflected calibration mismatch. The mismatch is effectively stable with time between the two channels as long as the configuration of antennas and connecting cables does not change. Calibration of the reflectometer consists of determining the ratio of the signal processing gain in the bottom channel to that in the top channel. Once established the ratio of the reported bottom channel "power" to 
that of the top channel can be scaled to give a gain-difference-corrected reflectivity (ratio of reflected power to incident power)

\subsubsection{Over-water calibration method}

During SMEX02, the GPS reflectometer was flown every day over a large body of water before and after data collection. This process allowed the observation of reflected power values corresponding to smooth-surface, high elevation reflectivity of water from several different satellites. The obtained water reflectivity was scaled to the value of 63 percent corresponding to the normalincidence reflectivity of water at L-band. Subsequently, signals reflected from the soil surface were referenced to the over-water calibrated signal using the scale factor determined in this way.

For research purposes, and with data obtained throughout the study period, the calibration stability was verified at numerous water-overpasses to account for satellite illumination variation and possible A/D drift. The averaged calibration factors are presented on Table 3 for different satellites (expressed as the satellite's PRN code) throughout the study period; the average calibration factor for all days was 1.72 .

\begin{tabular}{|c|c|c|c|}
\hline Date & Satellite PRN & Calibration Factor & Daily Avg \\
\hline $6 / 25 / 2002$ & 24 & 1.65 & \multirow[b]{3}{*}{1.67} \\
\hline $6 / 25 / 2002$ & 23 & 2.00 & \\
\hline $6 / 25 / 2002$ & 10 & 1.35 & \\
\hline $6 / 27 / 2002$ & 24 & 1.50 & \multirow[b]{2}{*}{1.65} \\
\hline $6 / 27 / 2002$ & 10 & 1.80 & \\
\hline $7 / 1 / 2002$ & 24 & 2.00 & \multirow[b]{2}{*}{1.98} \\
\hline $7 / 1 / 2002$ & 10 & 1.95 & \\
\hline $7 / 5 / 2002$ & 24 & 1.85 & \multirow[b]{3}{*}{1.65} \\
\hline $7 / 5 / 2002$ & 23 & 1.53 & \\
\hline $7 / 5 / 2002$ & 10 & 1.58 & \\
\hline $7 / 8 / 2002$ & 24 & 1.70 & \multirow[b]{3}{*}{1.72} \\
\hline $7 / 8 / 2002$ & 23 & 1.70 & \\
\hline $7 / 8 / 2002$ & 10 & 1.75 & \\
\hline \multicolumn{2}{|c|}{ Average } & 1.72 & \\
\hline
\end{tabular}

Table 3. Over-water calibration factors used for SMEX02 GPS data calibration.

\subsubsection{Direct-signal calibration method}

During all SMEX02 data taking intervals (approximately two hours,) the direct signal was subject to constant modulation due to multi-path interference. According to the GPS specification document, the illumination power of the 
satellites varies slowly with elevation angle, so the GPS illumination can be considered constant for short time intervals. It was assumed then that the signal variation due to orbital changes was much smoother than the fluctuations observed, so that the existing modulation was attributed to multi-path interference.

The acquired direct signal could not be used directly as a normalizing factor for the reflected data; the interference incorrectly changed the observed reflectivity so that it did not correspond to surface features. Therefore, the direct signal was modeled by fitting the raw direct data to a third order polynomial. The polynomial function was assumed to be a good approximation of the relatively constant source of GPS illumination absent multi-path effects.

\subsection{Determining total scattered signal power}

Estimation of surface reflectivity also required a correct interpretation of the reflected power. Two methods were implemented to relate the collected data to surface features. First, reflections from specular areas were assumed over the relatively flat Walnut Creek region (see Figure 3.) The second method utilized the surface slope probability density and the acquired data to obtain another estimate of surface reflectivity.

\subsubsection{Signal power from specular locations}

If small irregularities on the surface are neglected and normal incidence is assumed, the first Fresnel zone is approximated by an ellipse with a semi-major axis of 14.5 meters. Note that the first half-code chip range bin extends to a semi-major axis of 567 meters. Thus, the first range bin easily encompasses the signals reflecting from the first Fresnel zone. In this case, plus or minus one-half code chip from the peak of the lambda-squared function represents the average power reflected from that area.

Nonetheless, the correlation in the receiver is usually not perfect, so that the peak of the lambda-squared function is slightly lower than what it should be. The true peak can be approximated by sampling the lambda-squared function at an arbitrary initial point for each half-code chip and reconstructing the waveform assuming the ideal shape. Estimation of the peak reflected signal, along with over-water and direct-signal calibration, were used to determine surface reflectivity.

\subsubsection{Compensation for surface topography effects}

For the case where the terrain cannot be assumed flat, the peak of the reflected power does not correctly represent the surface reflectivity. In this case, the 
reflected signal correlation function is broadened by contributions from signals outside the first Fresnel zone that are observed at delays greater than one halfcode chip.

As described earlier Katzberg and Garrison (1996) show that the signal recorded by the reflectometer can well be modeled as the result of the convolution of the ideal lambda-squared profile with the slope probability density function of the surface. Consequently, in the case of a rough surface, the correct representation of the surface reflectivity requires a compensation for this process using the collected data and some knowledge on surface topography. In other words, surface scattering causes the incident power to be distributed more or less over a range of delays. The power versus delay must be reconstituted so that the only residual effect is surface reflection coefficient. In effect the scattering probability density must be integrated to arrive at the multiplying factor, the reflection coefficient.

The roughness effects were accounted for by modeling the surface slopes as two dimensional, Gaussian distributed, random variables with isotropic mean square slope. Inferring the associated parameters is done by comparing a set of model power versus delay waveforms with the recorded data. The model waveforms come from the ideal lambda-squared waveform convolved with the twodimensional Gaussian surface slope model. The best match allows the mean square slope and amplitude of the measured data to be determined. The result provided an approximation of the reflectivity from a larger spatial resolution cell than for the specular approach (e.g. 567 meters.) If the scattering is over a small band of angles the specular assumption and this probability reconstitution approach are expected to yield similar results. At the same time, differences in extracted reflectivity are good indicators of surface areas in which the reflectivity extraction is suspect.

Justification for the reasonableness of the two approaches is given using Figure 3 , which shows the mean slope taken at the Walnut Creek sites over a large number of sample points. While there are a few peaks in the data, the slopes tend to remain under 10 degrees, with the bulk of the slopes around 5 degrees. One half code chip range bin at 1100 meters altitude covers a scattering angle close to 13 degrees, making it likely to capture the energy from virtually all slopes within a fraction of a code chip. Thus the recorded data is expected to produce a power versus delay similar to the flat surface (specular) condition (See figure 4 and the associated discussion.) 




Figure 3. Mean slopes taken from several of the Walnut Creek sites.

\section{Processing and Results}

\subsection{Reflectivity extraction}

The GPS reflectometer provided three general sets of data: the direct, the reflected, and the navigation data. The computation of surface reflectivity and correlation with ground features required the integration of the three data sets based on the time of data collection.

The data provided by the navigation file was used to find the location of the reflectometer based on the WGS-84 ellipsoid (latitude, longitude, and elevation) as well as the location of the satellite with respect to the receiver (elevation and azimuth angles.) The aircraft altitude was further corrected using a local digital elevation model (DEM) to properly compute the location of the specular points with equation 3.

The reflected and direct data were calibrated using the methods described in sections 3.2.1 and 3.2.2, respectively. Subsequently, the reflected data were processed to find the correct amplitude of the lambda-squared function via the "specular assumption" and "probability reconstitution" methods (as explained in § 
3.3.1 and 3.3.2.) The resulting reflected data were normalized using the calibrated direct signal to yield surface reflectivity. Furthermore, the vegetation canopy effects were approximated and removed from the reflectivity estimates using in-situ data as input into equation 4 . The dielectric loss factor was obtained from a model presented in Ulaby et al. (1986) as a function of the volumetric moisture in the leaves at $1.5 \mathrm{GHz}$.

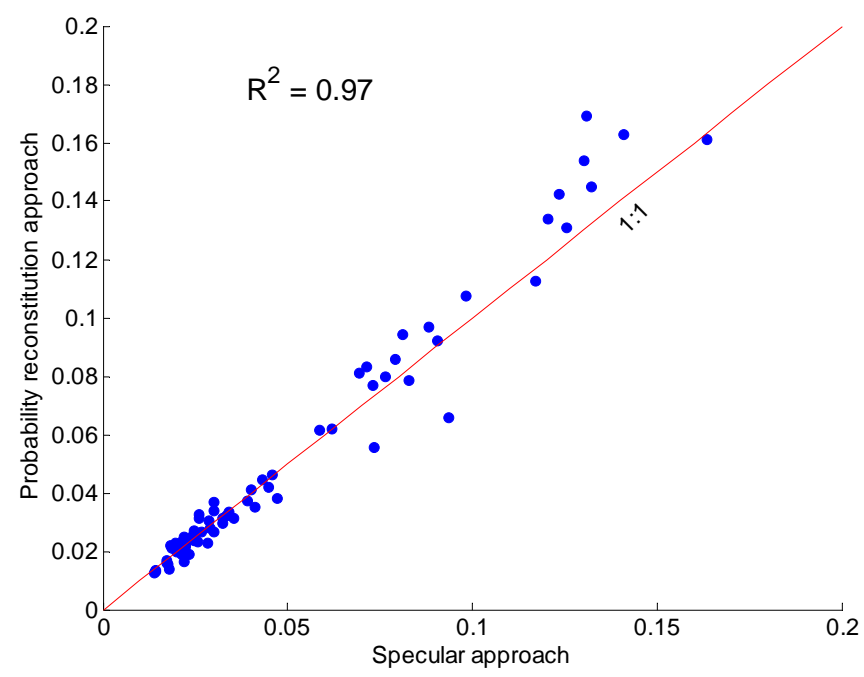

Figure 4. The reflectivity estimation via both the specular approach and the probability reconstitution method yielded similar results.

Except for a few cases, the reflectivity obtained with the probability reconstitution approach yielded similar values to those obtained by the specular approach (Figure 4.) Note that scattering from leaves and stalks would manifest itself as a deviation from the 1:1 slope in Figure 4 in support of the conclusion that attenuation was the dominant effect from biomass.

Typical results of this processing are shown in Figure 5, which shows two images of reflectivity, one before and one after the July $4^{\text {th }}$ rain. The images represent plots of the reflectivity at the specular points geo-located on a panchromatic Landsat7 ETM image taken on July $1^{\text {st }} 2002$. The plots show a qualitative correlation of the reflected data with high-reflectivity surface features such as rivers, lakes, ponds, et cetera (water bodies appear dark in the Landsat image because of the strong near-IR absorption of water.) The color code is such that yellow represents reflectivity greater than 26 percent while dark red represents reflectivity less than 2 percent. The two images demonstrate a high contrast between the reflectivity of the dry land (left image,) and that of wet land (right image.) No noticeable modulation was observed in the raster-like reflectivity pattern as it would be if calibration errors were present due to multi-path or due to power differences from a change of tracked satellite [Masters, et al. 2004]. Note that signals from satellites with PRN 10, 23, and 24 are plotted in Figure 5. 
A more detailed look at the reflectivity data, day-to-day, over the lake areas showed the reflectivity close to that of water (63 percent,) regardless of which satellite data were used.
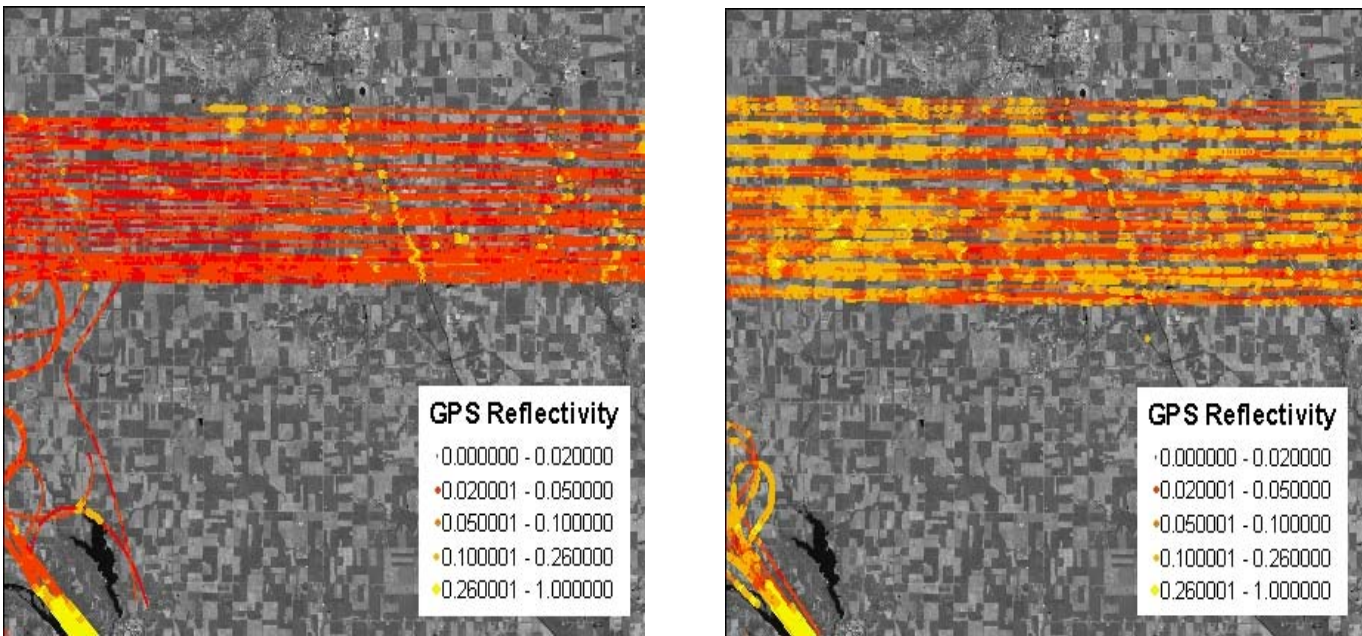

Figure 5. The left panel (a) illustrates reflectivity values from aircraft tracks done on July $1^{\text {st }}$ after approximately ten days without rain. The right panel (b) represents reflectivity data from July $5^{\text {th }}$, approximately 12 hours after rain. (Note that the scales are the same for reflectivity and that the values over the lake in the bottom left corner are the same.)

\subsection{Dielectric constant comparison with in-situ data}

In order to relate the reflected GPS to surface geophysical parameters a comparison with predicted dielectric constant was chosen. The fundamental parameter controlling the interaction of the microwave signal with the surface is the dielectric constant. Previous work with remote sensing instruments has utilized the dielectric constant as a unifying principle among the remote sensing techniques and considerable effort has gone into the development of dielectric models for the soil. In this section, compensation for each of the known influences on dielectric constant is presented based on in-situ data from SMEX02 including surface roughness, vegetation canopy and soil moisture effects.

To extract the dielectric constant, the GPS reflectivity was converted to an estimated surface dielectric constant by solving for $n_{2}$ from the Fresnel equation for normal incidence,

$$
\text { (5) } \quad R_{1,2}=\left|\frac{n_{1}-n_{2}}{n_{1}+n_{2}}\right|^{2} \text {, }
$$

where $R_{1,2}$ is the reflectivity for both horizontal and vertical polarizations, and $n_{1}$ and $n_{2}$ refer to the refractive index of air and soil respectively (where $n \cong \sqrt{ } \varepsilon$.) Use of the near normal condition is justified based on the fact that the GPS reflectometer was programmed to select only satellites above 55 degrees with 
the data taken generally above 65 degrees. For the nearly elliptically polarized reflected signal, it was verified that the error in using the vertical value (equation 5 ) was only five percent of the reflectivity at the worst-case elevation angle.

The in-situ soil moisture data were converted to surface dielectric constant values using the Wang-Schmugge model. This model was selected because it explicitly takes into account free and bound water (details of the model are presented by Wang and Schmugge, 1980.) The GPS derived surface dielectric constant and the theoretical surface dielectric constant were compared keeping in mind that the imaginary component of the soil dielectric constant $\varepsilon^{\prime \prime}$ is negligible at GPS frequencies [Ulaby et al., 1986].

The dielectric constant estimated from the GPS data over corn and soybeans is shown in Figure 6 as a function of volumetric soil moisture derived from Theta probe measurements. The dielectric constant estimated using the WangSchmugge model is also plotted for comparison purposes. It is clear from Figure 6 that the GPS dielectric constant underestimates the modeled dielectric constant. In addition, the dielectric constant over cornfields is noticeably noisier than the dielectric constant over soybeans: variance over corn is approximately 6.44 while the variance over soybeans is approximately 2.52 .
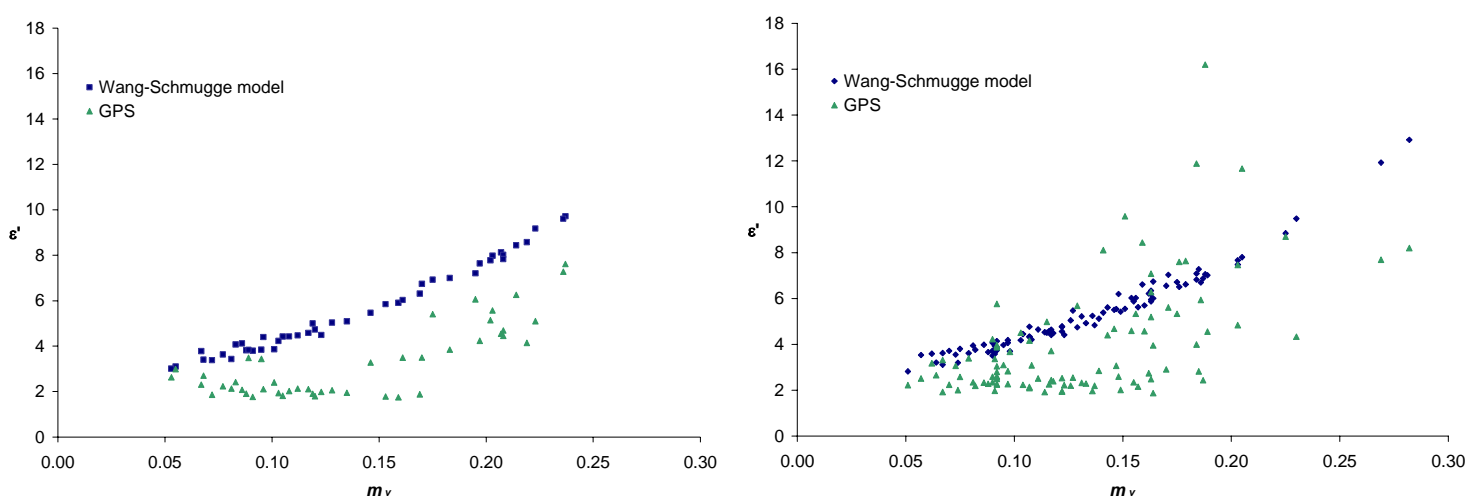

Figure 6. GPS-estimated dielectric constant over (a) soybeans and (b) corn as a function of soil moisture from Theta probe measurements.

Biomass attenuation effects were observed during comparison of GPS-estimated and the modeled dielectric constant. Figure 7 a shows the percent error of the estimated dielectric constant without accounting for any vegetation effects; the percent error is the absolute difference between the GPS-derived and the modeled dielectric constant. Vegetation attenuation was accounted for through equation 4; the value for $\varepsilon$ " was approximated at 17 by assuming the plant leaves were under no stress and that they were at or near maximum water content as described in page 2110 of Ulaby et al. (1986). After attenuation was accounted for through equation 4 (Figure $7 \mathrm{~b}$,) the mean error decreased from $54.65 \%$ to $38.27 \%$, but the standard deviation increased from $12.10 \%$ to $20.25 \%$. 

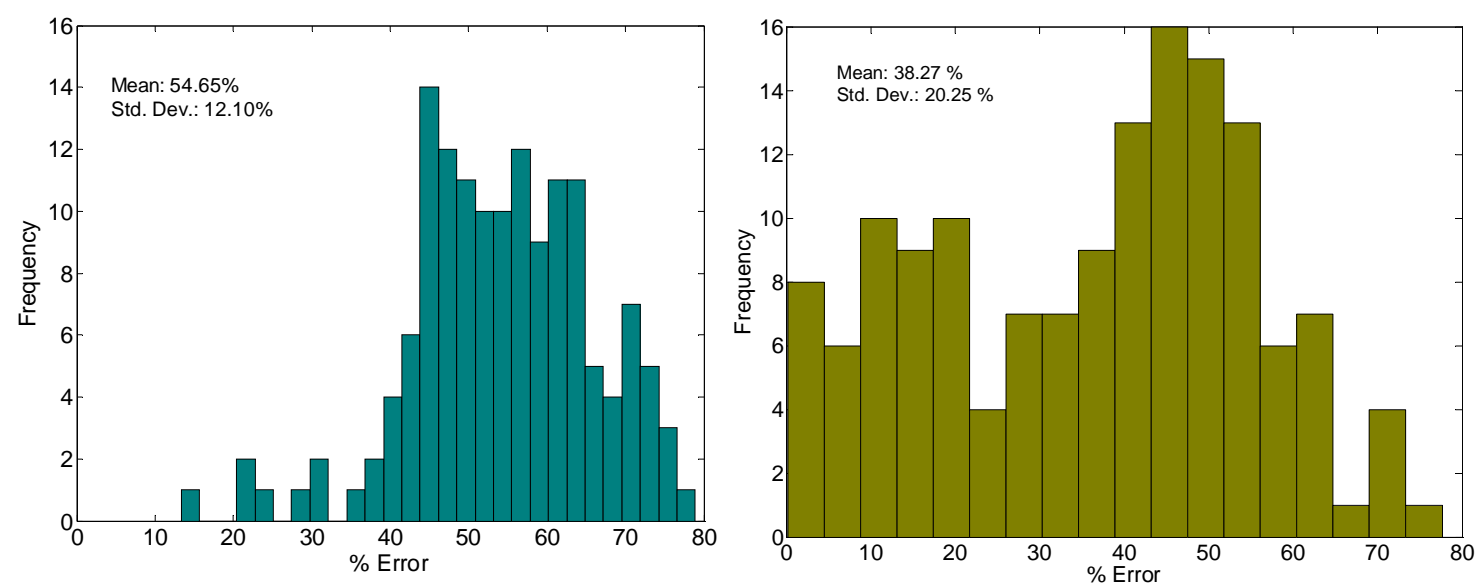

Figure 7. Percent error between the GPS and the modeled dielectric constant (from Theta probe measurements:) (a) the left panel shows the error without considering vegetation attenuation while (b) the right panel shows the error considering vegetation effects.

Another comparison was done using the soil moisture values from the top $1 \mathrm{~cm}$ of soil at the four sites where these data were collected. Before the rain on July $4^{\text {th }}$, the soil moisture readings from the top $1 \mathrm{~cm}$ layer of soil were, on average, $40 \%$ lower than those measured via the Theta probe. After the July $4^{\text {th }}$ rain, the Theta probe readings were lower than those of the core samples but the two measurements were in close agreement (Figure 8.) Since soil moisture readings at the top $1 \mathrm{~cm}$ of soil were only collected at four sites while theta probe readings were collected across the region, the Theta probe measurements taken before July $4^{\text {th }}$ were decreased by a factor of 0.4 to model the dry conditions at the surface. After the rain, Theta probe readings were used for the moisture values of the top-most soil layer without modification. This method provided an estimation of the soil moisture of the top $1 \mathrm{~cm}$ of soil across the region which was used as input to the Wang-Schmugge model.
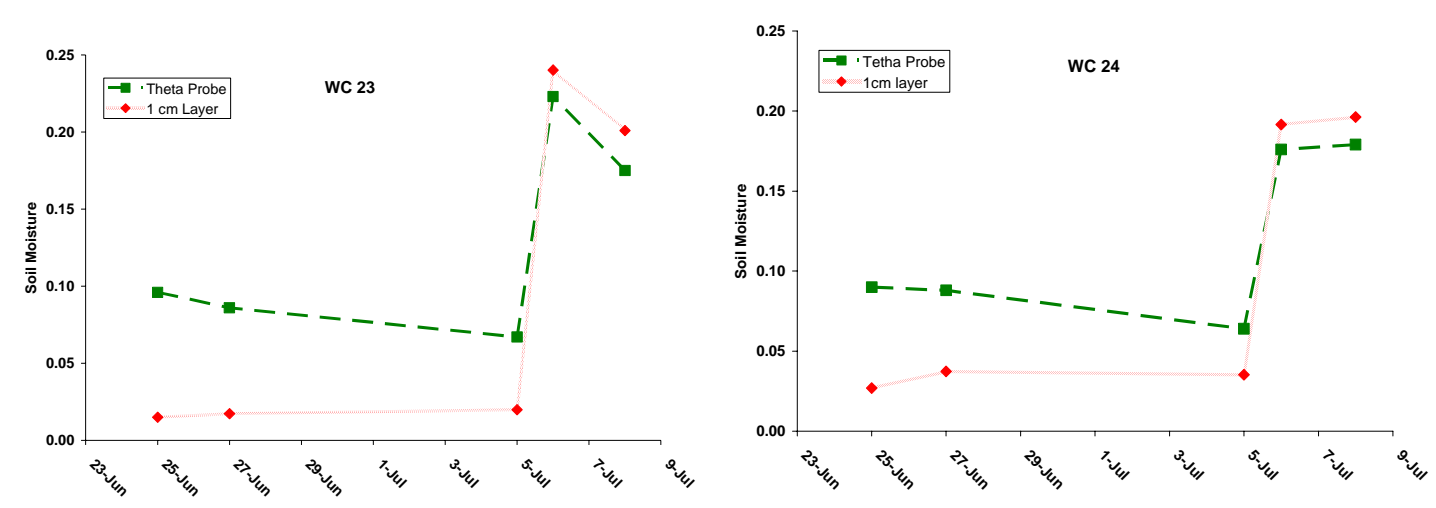

Figure 8. Before the rain, core sample measurements show lower soil moisture conditions at the top $1 \mathrm{~cm}$ layer than the Theta probe $5-\mathrm{cm}$ average. After the rain, both measurements are in closer agreement. 
As illustrated in the histogram in Figure 9a, the measured versus theoretical data had a decreased percent error of $46.58 \%$ and a standard deviation of $11.21 \%$ when vegetation was neglected. Figure $9 \mathrm{~b}$ shows that considering the attenuation due to leaves yielded a mean percent error of $28.54 \%$ and a standard deviation of $16.73 \%$. Figure 10 shows the dielectric constant over both crops, using the soil moisture from the top $1 \mathrm{~cm}$ of soil as the independent variable. The result is closer agreement between the GPS-estimated and the modeled dielectric constant.
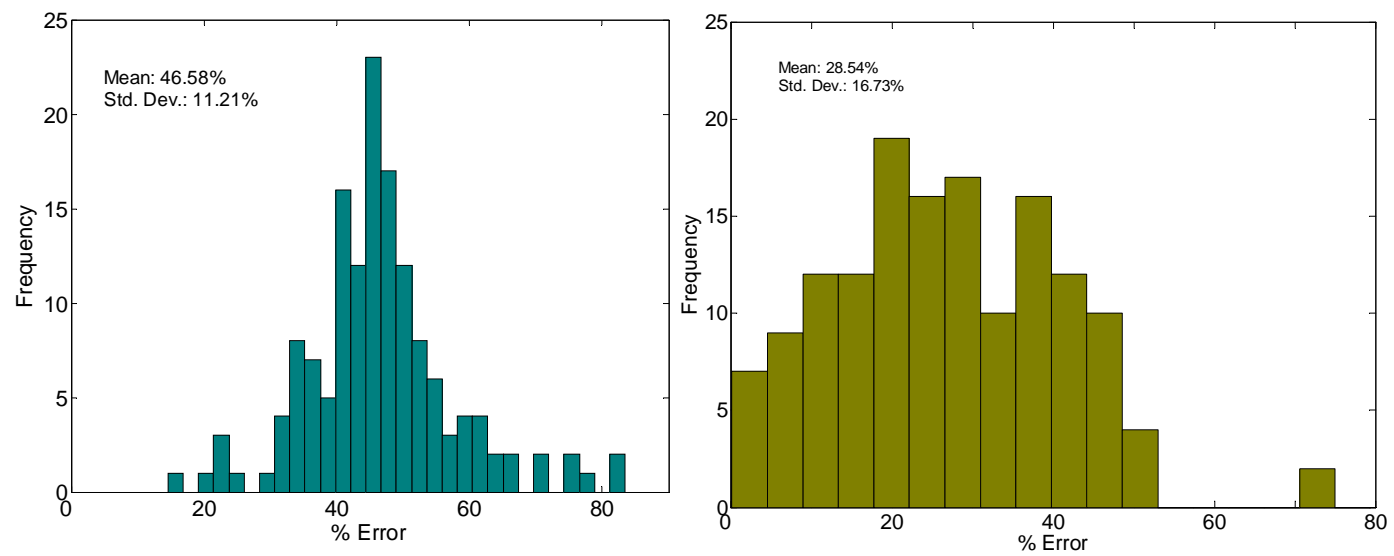

Figure 9. Comparing GPS-estimated to modeled dielectric constant from the top-most layer of the soil yields a reduction in the mean percent error when (a) neglecting vegetation attenuation and (b) when considering it.

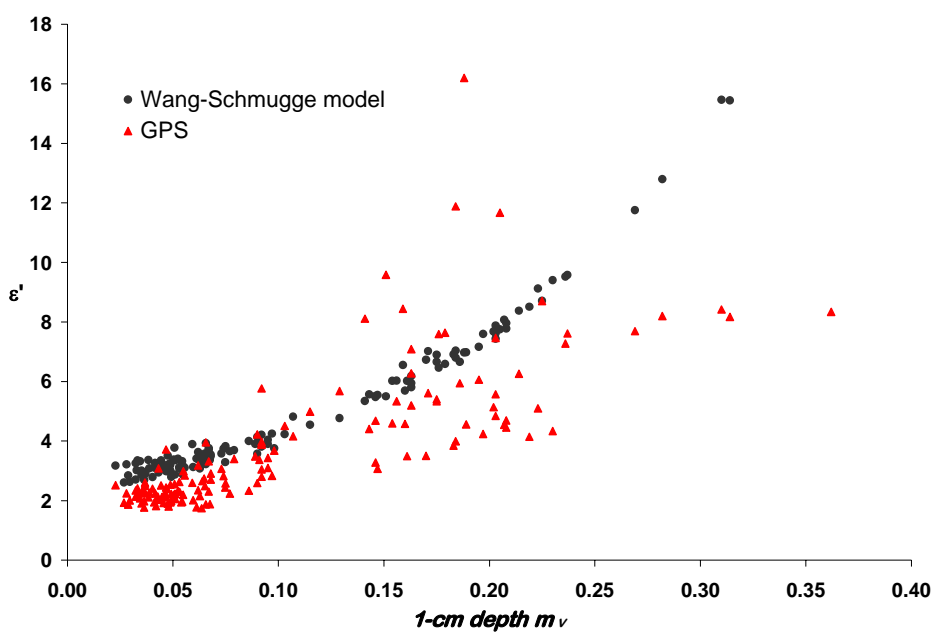

Figure 10. Measured and theoretical dielectric constant over both crop types compared to soil moisture values at the top-most layer of soil.

\section{Discussion}


Good agreement between GPS-derived and modeled dielectric constant under a variety of precipitation conditions has demonstrated the effectiveness of the processing methods used in this study. The direct signal smoothing process, along with the relative stability between the direct and reflected signal channels, produces a stable, repeatable system calibration. Multipath effects that limit the utility of the normalization of the reflected signals are greatly reduced by the calibration of the direct data. Figure 5 shows the stability of the calibration; the reflectivity remains regularly low over dry soil moisture conditions and increases rapidly over bodies of water. Using the calibrated signal, fluctuations in reflectivity not related to surface features are greatly reduced. The GPS remote sensing technique provides a practical tool for monitoring regional soil moisture and distribution of precipitation.

The observed underestimation of dielectric constant due to measured low reflectivity is an issue that requires further investigation to resolve. Effects due to vegetation attenuation and surface scattering were considered, which reduced the error between the GPS-estimated dielectric constant and that of the numerical model. The removal of vegetation attenuation increased the mean accuracy by $30 \%$ (when using Theta probe measurements,) while the precision was reduced by $67 \%$. Better agreement was observed between theoretical predictions and experimental data when soil moisture values from the top $1 \mathrm{~cm}$ layer of soil were used; the minimum mean error was estimated at $28.54 \%$.

The comparison of GPS data with the top $1 \mathrm{~cm}$ layer of soil sheds light on the issue of sensing depth. The soil density and soil moisture profiles as functions of depth are major controlling factors for effective sensing depth of the GPS technique. The fact that surface moisture was low and increased with depth before the advent of rain suggests the possibility of an anti-reflection coating-like effect from the surface. As is well-known, the existence of a layer of material of intermediate dielectric constant in a sandwich configuration can cause a much reduced reflectivity [Born and Wolf 1964]. It is not known if this or a related effect explains the observed discrepancies but suggests the need for further investigation.

Other possible causes of the discrepancies were investigated during the course of this study. Effects of soil conductivity, possible receiver miscorrelation, validity of models and imperfect circular polarization were investigated and none were found to yield significant contributions to the observed differences between experimental and theoretical data. In addition, the effectiveness of utilizing the biomass attenuation approach was investigated by comparing reflectivity estimates from corn at early and late stages of growth (medium to high biomass) with soybeans at low to medium biomass. No difference in surface reflectivities was found, attesting to the effectiveness of using equation 4 . However it was noted that in this work and the previous report [Masters, et al., 2004] that the reflectivity of corn fields showed considerably more scatter than that of soy beans. Nonetheless, the reflectivity obtained using the two approaches 
described previously and presented in Figure 4 show the minimal impact of leaf scattering supporting theory that suggest that leaf absorption is far more significant.

\section{Conclusion}

In summary, this study has shown that the land-reflected GPS signal can be readily detected and possibly serve for observations of regional soil moisture distribution. The relationship of the GPS reflected signal (i.e. reflectivity) to soil dielectric constant and to volume soil moisture was investigated. A method of GPS reflectivity calibration involving both the direct signal and the over-water value of reflected signals has been presented. Using soil moisture profiling data from available locations, soil moisture in the top $1 \mathrm{~cm}$ of soil was inferred from the $5 \mathrm{~cm}$ average Theta probe measurements taken over the entire SMEX02 study area. Soil moisture values for the top $1 \mathrm{~cm}$ of the soil were more closely correlated to (calibrated) GPS reflectivity than were the $5 \mathrm{~cm}$ average Theta probe readings. Overall, the dielectric constant derived from GPS data compared well to the Wang-Schmugge theoretical curve, but was generally lower at all values of soil moisture found in the SMEX02 data. The effect of signal attenuation due to vegetation cover was taken into consideration, and a number of possible causes for the remaining discrepancy between experimental and theoretical (model-derived) data were investigated. These include the possibility of an anti-reflection coating effect on the soil surface.

\section{Appendix A: Theta probe functionality}

Theta Probes consist of a waterproof housing that contains the electronics, a coaxial cable, and a probe fashioned from four sharpened stainless steel rods that are inserted into the soil. The Theta probe electronics generates a $100 \mathrm{MHz}$ sinusoidal signal, which is applied to a specially designed internal transmission line that extends into the soil by means of the array of four rods. The impedance of this array varies with the impedance of the soil, which has two components the apparent dielectric constant and the ionic conductivity. A voltage standing wave is set up in the coaxial line feeding the probe head. The voltage standing wave ratio (VSWR) is affected by the dielectric properties of the material into which the soil is inserted. Shifts in the location of voltage-standing-wave ratio nulls can be interpreted in terms of the dielectric constant of the soil. Because the dielectric constant of water is very much higher than the dielectric constant of either soil or air, the dielectric constant of soil is dominated by its water content. The output signal covers a range of soil dielectric constants, $\varepsilon$, between 1 and 32 , corresponding to approximately $0.0-0.5 \mathrm{~m}^{3}-\mathrm{m}^{-3}$ in volumetric moisture content for all soil types found in the Walnut Creek study area.

Since the probe is only a small fraction of a wavelength at the device's operating 
frequency, the effective capacitance of the probe is the integral of the dielectric constant along the length of the probe. The probe's output is then expected to respond to the average dielectric constant along its length and not be more sensitive to one end or the other.

The response of the Theta probe to the average dielectric constant must be considered when interpreting the results. The penetration of the microwave signal into the soil can be as low as $\lambda_{0} / 10$ [Ulaby et al., 1986] and the comparison between the observed GPS reflectivity and the Theta probe reported values must take this into consideration.

\section{References}

Auber, J., Bibaut, A., R, J. (1994). Characterization of Multipath on Land and Sea at GPS Frequencies. Proc. $7^{\text {th }}$ International technical Meeting Satellite Division of Institute of Navigation, Part 2, ION GPS-94, pp. 1155-1171.

Born, M., and Wolf, E. (1964). Principles of Optics, London: Pergamon Press, pp. 59-66.

Beckmann, P., and Spizzichino, A. (1963). The Scattering of Electromagnetic Waves from Rough Surfaces, New York: Pergamon Press,

Dobson, M.C., F. T. Ulaby, et al. (1985), Microwave Dielectric Behavior of Wet Soil-Part II: Dielectric Mixing Models, IEEE Transactions of Geoscience and Remote Sensing, ge-23 no. 1, 35-46.

Garrison, J. L., Komjathy, A., Zavorotny, V. U., Katzberg, S. J. (2002). Wind Speed Measurement Using Forward Scattered GPS Signals. Trans. Geosci. Remote Sens. Vol. 40, No. 1, pp. 50-65.

Garrison, J. L., Katzberg, S. J., Hill, M. I. (1998). Effects of sea roughness on bistatically scattered range coded signals from the Global Positioning System. Geophys. Res. Let. Vol. 25, No. 13, pp 2257-2260.

Hegarty, C., Kim, T., Ericson, S., Reddan, P., Morrissey, T., Van Dierendonck, A. J., (1999). Methodology for Determining Compatibility of GPS L5 with Existing Systems and Preliminary Results, Proc. Annual Meeting Institute of Navigation.

Holmes, T. (2003). Measuring surface soil parameters using passive microwave remote sensing. The ELBARA campaign 2003, HD-No.: 639. Vrije Universiteit Amsterdam.

Jackson, T.J., et al. (2002), Soil Moisture Experiments in 2002, Subtitled 
"Experiment Plan, June 2002", http://hydrolab.arsusda.gov/smex02/

Jackson, T. J., Le Vine, D. M., Swift, C. T., Schmugge, T. J. (1994). Large Area Mapping of Soil Moisture Using the ESTAR Passive Microwave Radiometer.

Proc. $6^{\text {th }}$ Int. Symposium on Physical Measurements and Signatures in Remote Sensing. pp. 467-474.

Jackson, T. J., and Schmugge, T. J. (1992). Surface Soil Moisture Measurement with Microwave Radiometry. $43^{\text {rd }}$ Congress of the International Astronautical Federation. IAF 92-0122.

Jackson, T. J., and O'Neil, P. E. (1988). Observed Effects of Soil Organic Matter Content on the Microwave Emissivity of Soils. Proc. Int. Remote Sensing Symposium. Vol. 2, pp. 673-676. Edinburgh, Scotland.

Katzberg, S. J., and Garrison J. L. (1996). Utilizing GPS to Determine Ionospheric Delay Over the Ocean, NASA Technical Memorandum 4750.

Kerr, Y. H., Waldteufel, P., et al. (2001). Soil Moisture Retrieval from Space: The Soil Moisture and Ocean Salinity (SMOS) Mission. IEEE Trans. Geosci. Remote Sens. Vol. 39, No. 8, pp. 1729-1735.

Koster, R. D., Dirmeyer P. A. (2004). Regions of Strong Coupling Between Soil Moisture and Precipitation. Science, Vol. 305, pp. 1138-1140

Kostov, K. G., Michalev, M. A., Nedeltchev, N. M., Gaidarova, S. G., Davidov, D. D., Spasov, A. Y., and Vichev, B. I. (1991). Bulgarian Effort Toward The Operational Use of Passive Microwave Remote Sensing of Soil Moisture. Proc. Int. Geosci. Remote Sens. Symposium. Vol. 2. pp. 749-751. Espoo, Finland.

Laymon, C. (2003). SMEX02 Sliced Core Soil Moisture Data, Walnut Creek Watershed, lowa. National Snow and Ice Data Center.

Le Vine, D.M., Griffis, A. J., Swift, C. T., and Jackson, T. J. (1994). ESTAR: A Synthetic Aperture Microwave Radiometer for Remote Sensing Applications. Proc. IEEE, vol. 82, no. 12, pp1787-1801.

Masters, D., Axelrad, A., Katzberg, S. J. (2004). Initial results of land-reflected GPS bistatic radar measurements in SMEX02. Remote Sensing of Environment. Vol. 92, pp. 507-520.

Narayan, U., Lakshmi, V., Njoku, E. G. (2004). Retrieval of soil moisture from passive and active L/A band sensor (PALS) observations during the Soil Moisture Experiment in 2002 (SMEX02). Remote Sensing of Environment. Vol. 92. pp. 483-496. 
Njoku, E. G., Lakshmi, V., O'Neil, P. E. (2004). Soil moisture field experiment special issue. Remote Sensing of Environment. Vol. 92, pp. 425-426.

Njoku, E. G. (1999). AMSR Land Surface Parameters, Algorithm theoretical Basis Document. Jet Propulsion Laboratory, California Institute of Technology, Pasadena, CA.

Njoku, E. G., and Kong, J. (1977). Theory for Passive Microwave Remote Sensing of Near-Surface Soil Moisture. J. Geophys. Res. Vol. 82. No. 20, pp. 3108-3118.

O'Neil, P. E., Jackson, T. J. and Chauhan N. S. (1993). Microwave Soil Mo Schmugge, T., T. J. Jackson, W.P. Kustas, and J. R. Wang (1992), Passive microwave remote sensing of soil moisture: results from HAPEX, FIFE and MONSOON 90, ISPRS Journal of Photogrammetry and Remote Sensing, 47, 127-143.

Spilker, J. J., Parkinson, B. W. (1996). Overview of GPS Operation and Design. In Global Positioning System: Theory and Applications Volume I (pp. 29-55). Washington, DC: American Institute of Aeronautics and Astronautics.

Torres, O. (2004), Analysis of Reflected Global Positioning System Signals as a Method for the Determination of Soil Moisture, Master's Thesis, The University of Texas at El Paso, El Paso, Texas.

Torres, O., Katzberg S. J. (2002). Analysis of reflected Global Positioning System (GPS) signals from land for soil moisture determination and topography mapping. Proc. Earth Observing Systems VII. Vol. 4814, pp. 9-15.

Ulaby, F.T., R. K. Moore, and A. K. Fung (1986). Microwave Remote Sensing: Active and Passive. Volume III From Theory to Applications. Norwood: Artech House.

Wang, J.R., and Schmugge, T. J. (1980). An Empirical Model for the Complex Dielectric Permittivity of Soils as a function of Water Content, IEEE Trans. Geosci. Remote Sens. GE-18, pp 288-295.

Wilson, W. J., Yueh, S. H., Dinardo, S. J., Chazanoff, S. L., Kitiyakara, A., Li, F. K., Rahmat-Samii, Y. (2001). Passive Active L- and S-Band (PALS) Microwave Sensor for Ocean Salinity and Soil Moisture Measurements. IEEE Trans. Geosci. Remote Sens. Col. 39, No. 5, pp. 1039-1048.

Wood, E. F., Lin, D. S., Mancini, M. Thongs, D., Troch, P. A., Jackson, T. J., Famiglietti, J. S., and Engman. E. T. (1993). Intercomparisons Between Passive and Active Microwave Remote Sensing, and Hydrological Modeling for Soil Moisture, Adv. Space Res. Vol. 13, No. 5, pp. (5)167-(5)176. 